\title{
Cisplatin induces HepG2 cell cycle arrest through targeting specific long noncoding RNAs and the p53 signaling pathway
}

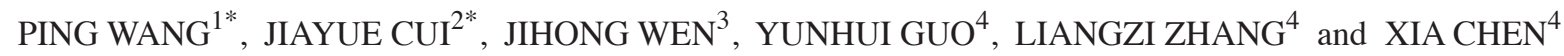 \\ ${ }^{1}$ Department of Otolaryngology-Head and Neck Surgery, First Hospital of Jilin University; \\ ${ }^{2}$ Department of Histology and Embryology, College of Basic Medical Sciences Jilin University; \\ ${ }^{3}$ Department of Gynaecology and Obstetrics, First Hospital of Jilin University; \\ ${ }^{4}$ Department of Pharmacology, College of Basic Medical Sciences, Jilin University, Changchun, Jilin 130021, P.R. China
}

Received May 14, 2015; Accepted September 28, 2016

DOI: $10.3892 / \mathrm{ol} .2016 .5288$

\begin{abstract}
Cisplatin has been used effectively in the treatment of hepatocellular carcinoma (HCC). Long noncoding RNAs (lncRNAs) were recently reported to contribute to the pathogenesis and progression of HCC. Their molecular mechanism related to cisplatin treatment remains unclear. The purpose of this study is to identify specific lncRNAs and to clarify their functions in HCC after cisplatin exposure. Reannotation and identification of differentially expressed lncRNAs were performed using the microarray data set GSE38122 in the Gene Expression Omnibus database. Four significantly differentially expressed lncRNAs (RP11-134G8.8, RP11-612B6.2, RP11-363E7.4 and RP1-193H18.2) were identified in HepG2 cells exposed to cisplatin by bioinformatics methods. The upregulated RP11-134G8.8 and RP11-363E7.4 and the downregulated RP1-193H18.2 were confirmed by reverse transcription-quantitative polymerase chain reaction. Furthermore, 57 significant co-expressing genes and their corresponding pathways were annotated and identified. The p53 signaling pathway showed the most significant difference among all pathways. Based on these results, the cell cycle and three key genes, cyclin-dependent kinase inhibitor 1A (CDKN1A, also known as p21), tumor protein p53 inducible protein 3 (TP53I3) and wild-type p53-induced phosphatase 1 (Wip1, also known as PPM1D), were examined. CDKN1A, TP53I3 and PPM1D were all downregulated by RP1-193H18.2 but upregulated by
\end{abstract}

Correspondence to: Professor Xia Chen, Department of Pharmacology, College of Basic Medical Sciences, Jilin University, 126 Xinmin Street, Changchun, Jilin 130021, P.R. China

E-mail: chenx@jlu.edu.cn

${ }^{*}$ Contributed equally

Abbreviations: HCC, hepatocellular carcinoma; lncRNA, long noncoding RNA

Key words: long noncoding RNA, cisplatin, hepatocellular carcinoma, p53 signaling pathway, drug targets
RP11-134G8.8 and RP11-363E7.4. And obvious S phase arrest was induced by cisplatin treatment for $24 \mathrm{~h}$ in HepG2 cells. Finally, the immunofluorescence results showed upregulation of TP53I3 and Wip1 and downregulation of p21 at the protein level. The results suggested that the lncRNAs RP11-134G8.8, RP11-363E7.4 and RP1-193H18.2, and their co-expression genes, which annotated into the p53 signaling pathway, could be potential targets for cisplatin treatment.

\section{Introduction}

Hepatocellular carcinoma (HCC) is the third leading cause of cancer mortality in the world, and the five-year survival rate is lower than 5\% (1). It has an unfavorable prognosis due to its spread, metastases and high rate of recurrence (2). Currently, treatment with platinum-based chemotherapy is one the main means of drug therapy for HCC. Cisplatin, the first generation of platinum drugs, is one of the most active anticancer chemotherapeutic drugs (3). As a cell cycle non-specific drug, it can inhibit effectively the proliferation of HCC by the cytotoxic effect $(4,5)$. The main targets of cisplatin are DNA, RNA and proteins with strong affinity to the nucleus (6). Cisplatin impairs DNA replication by producing DNA intra-strand cross-links and cisplatin-DNA complexes, or by binding to nuclear and cytoplasmic proteins $(7,8)$. Thus, it is primarily considered as a DNA-damaging anticancer drug $(5,6)$. However, the precise regulatory mechanisms by which cisplatin induces apoptosis and inhibits proliferation are not completely clear.

Recently, accumulating evidences suggested that long noncoding RNAs (lncRNAs) have many biological functions, such as regulation of transcription, modulation of nuclear structure and function, carcinogenesis and cancer progression (9-11). lncRNAs are RNA molecules $>200$-bp long without protein coding functions (7). IncRNAs, as epigenetic regulators, are also associated with chemotherapy sensitivity in cancers $(8,12)$. The lnRNA H19 was found to induce multidrug resistance 1 (MDR1)-associated drug resistance in liver cancer cells through regulation of MDR1 promoter methylation (13). In addition, the lncRNA H19 was markedly upregulated in liver cancer, while metastasis associated lung adenocarcinoma transcript 1 was upregulated in human and murine $\mathrm{HCC}$, and the HOX transcript antisense RNA (HOTAIR) levels increased 
in human HCC and a liver cancer cell line (14). Therefore, lncRNAs may serve as biomarkers for treatment response in cancers.

Furthermore, it was suggested that IncRNAs may play an important role in regulating gene expression (10). The functions of lncRNAs are mainly carried out by their secondary structure; however, this is difficult to decipher (15). Due to the considerable challenge of exploring the lncRNAs functions, a co-expression-based method has been developed, in which IncRNA functions are predicted based on the functions of their co-expressed protein-coding genes (16). Genes that have similar expression patterns under multiple conditions tend to be involved in the same pathways. The co-expressed protein-coding genes are potentially regulated by the corresponding lncRNAs.

Taken together, some IncRNAs would be involved in antitumor effects and/or resistance to cisplatin by regulating gene expression. In this study, we investigated the differential expression of lncRNAs in HepG2 cells at different times of cisplatin exposure. Four differentially expressed lncRNAs were identified and their co-expressing genes were obtained. The aim of the present study is to identify lncRNAs that may be valuable biomarkers of cisplatin-based functions and chemoresponse, and also candidates for therapy targets in HCC.

\section{Materials and methods}

Cell culture and main reagents. HepG2 (a human HCC cell line) was purchased from the Institute of Biochemistry and Cell Biology, Chinese Academy of Sciences (Shanghai, China). Cells were maintained in RPMI 1640 medium (Invitrogen; Thermo Fisher Scientific, Inc., Waltham, MA, USA) with 10\% fetal calf serum (Invitrogen; Thermo Fisher Scientific, Inc.), 100 U/ml penicillin (Sigma-Aldrich; Merck Milipore, Darmstadt, Germany) and $100 \mu \mathrm{g} / \mathrm{ml}$ streptomycin (Sigma-Aldrich; Merck Milipore) at $37^{\circ} \mathrm{C}, 5 \% \mathrm{CO}_{2}$. The Cell Cycle Detection kit was purchased from Beyotime Institute of Biotechnology (Suzhou, China). Anti-p21 [also known as cyclin-dependent kinase inhibitor 1A (CDKN1A)] (bs-10129R), anti-tumor protein p53 inducible protein 3 (TP53I3) (bs-6144R) and anti-wild-type p53-induced phosphatase 1 (Wip1, also known as PPM1D) (bs-2447R) antibodies were purchased from Beijing Biosynthesis Biotechnology Co., Ltd. (Beijing, China). The RNeasy Mini kit was purchased from Qiagen GmbH (Hilden, Germany), while First-strand cDNA Synthesis kit was purchased from Tiangen Biotech Co., Ltd. (Beijing, China). The LightCycler DNA Master SYBR Green I kit was purchased from Roche Diagnostics GmbH (Mannheim, Germany).

Microarray data set. Microarray data from the microarray data set GSE38122 (http://www.ncbi.nlm.nih.gov/geo/query/acc. cgi?acc=GSE38122) was collected from the Gene Expression Omnibus database. This study investigated differential gene expression in the messenger RNA (mRNA) content of the HepG2 cell line upon 12, 24 and $48 \mathrm{~h}$ of exposure to $7 \mu \mathrm{M}$ cisplatin (Sigma-Aldrich; Merck Milipore) and its solvent. In a total of 18 arrays, three biological replicates were performed per compound/solvent at three time points. The samples were examined with GeneChip ${ }^{\circledR}$ Human Genome U133 Plus 2.0 (HG-U133 Plus 2.0) Array from Affymetrix, Inc. (Santa Clara, CA, USA).

Reannotation and identification of differentially expressed IncRNAs. The probes on the HG-U133 Plus 2.0 array were reannotated for human IncRNAs using noncoding RNA function annotation server (ncFANs), as showed in its website (15). Differentially expressed lncRNAs were identified by the fold-change method (17). The lncRNAs with a fold-change value of $>2.0$ or $<0.5$ were considered as differentially expressed IncRNAs.

Obtaining co-expressing genes for each differentially expressed lncRNA. To obtain co-expressing genes for each differentially expressed lncRNA, we calculated the Pearson's correlation coefficient (PCC) between each differentially expressed lncRNA and all the genes across all 18 samples. The genes with a strict cut-off (PCC $>0.9$ or $<-0.9$ ) were identified as co-expressing genes. Then, pathway enrichment was implemented to identify the affected pathways of lncRNA co-expressing genes using Database for Annotation, Visualization and Integrated Discovery (DAVID) 6.7 (18).

Confirmation of differentially expressed lncRNAs by reverse transcription-quantitative polymerase chain reaction ( $q P C R)$. HepG2 cells were harvested at 3, 6, 12 and $24 \mathrm{~h}$ after cisplatin exposure $(7 \mu \mathrm{M})$. Total RNA was extracted and purified from HepG2 cells by RNeasy Mini kit (Qiagen $\mathrm{GmbH}$ ). Complementary DNA was obtained using First-strand cDNA Synthesis kit (Tiangen Biotech Co., Ltd.). qPCR was performed on an ABI StepOnePlus Real-Time PCR System (Applied Biosystems; Thermo Fisher Scientific, Inc.) using the LightCycler DNA Master SYBR Green I kit (Roche Diagnostics GmbH). The primer pairs were synthesized by Sangon Biotech Co., Ltd. (Shanghai, China). The primers used for qPCR had the following sequences: ENSG00000224818 sense, 5'-CTC TGGAGGGAGCAAGGA-3' and antisense, 5'-TGGACTCTG AGGGACTGG-3'; ENSG00000256185 sense, 5'-GGCACT TTTCAGAACATC-3' and antisense, 5'-TGTCGTGTATCA CAGCAT-3'; ENSG00000260912 sense, 5'-CGACCACCT ATTCCACTT-3' and antisense, 5'-GCCAGGAAGGCTCAA ATC-3'; and ENSG00000267194 sense, 5'-AAAACCCAC CTCCAGCAC-3' and antisense, 5'-GCGGCAATCCGTAAA GAA-3'. The PCR conditions were as follows: $95^{\circ} \mathrm{C}$ for $5 \mathrm{~min}$, followed by 30 cycles of $94^{\circ} \mathrm{C}$ for $30 \mathrm{sec}, 55^{\circ} \mathrm{C}$ for $30 \mathrm{sec}$ and $72^{\circ} \mathrm{C}$ for $30 \mathrm{sec}$, and then $72^{\circ} \mathrm{C}$ for $5 \mathrm{~min}$. Fluorescence values were collected, using the GADPH RNA expression level as internal control. The relative content of mRNA was calculated according to the following formula: Fold-change $=2^{-\Delta(\Delta \mathrm{Cq})}$, where $\mathrm{Cq}$ is the quantification cycle, $\Delta \mathrm{Cq}=\mathrm{Cq}_{(\text {target) }}-\mathrm{Cq}_{(\mathrm{GAPDH})}$ and $\Delta(\Delta \mathrm{Cq})=\Delta \mathrm{Cq}_{\text {(rreated) }^{-}}-\Delta \mathrm{Cq}_{\text {(untreated) }}$.

Cell cycle analysis. Cells were seeded into 6-well plates at $1 \times 10^{6}$ cells $/ \mathrm{ml}$ for $24 \mathrm{~h}$ and divided into two groups: Control group (untreated HepG2 cells) and cisplatin treatment HepG2 group $(7 \mu \mathrm{M})$. Cells were harvested, washed with PBS twice, centrifuged at room temperature (RT) for $10 \mathrm{~min}$ at $600 \mathrm{x} g$, resuspended in PBS and fixed in ice-cold $70 \%$ ethanol overnight at $4^{\circ} \mathrm{C}$. Cells were washed and resuspended with PBS, 
stained with propidium iodide for $30 \mathrm{~min}$ at $37^{\circ} \mathrm{C}$ in the dark, and then analyzed on a flow cytometer with the Cell Cycle Detection kit (Beyotime Institute of Biotechnology). A BD FACSAria flow cytometer (BD Biosciences, Franklin Lakes, NJ, USA) was used, and the data were analyzed using the QuantiCALC software version 1.0 (BD Biosciences).

Immunofluorescence and microscopic analysis. HepG2 cells were cultured on circular cover slips and treated with cisplatin $(7 \mu \mathrm{M})$ for 12 and $24 \mathrm{~h}$. Then, cells were washed with Hank's solution, fixed with $4 \%$ paraformaldehyde solution for $30 \mathrm{~min}$ at RT, and permeabilized with $0.1 \%$ Triton X-100 (dissolved in PBS) for 10 min. Next, cells were washed with PBS three times and blocked in $0.1 \%$ Triton X-100 [dissolved in PBS with 5\% goat serum (Invitrogen; Thermo Fisher Scientific, Inc.)] for $1 \mathrm{~h}$ at RT. After blocking, cells were incubated with $50 \mu$ l anti-TP53I3 antibody (1:100), anti-Wip1 antibody (1:200) or anti-p21 antibody $(1: 100)$ at $4^{\circ} \mathrm{C}$ overnight. Then, cells were washed three times with $\mathrm{PBS}$, and incubated with Alexa 555-conjugated secondary antibodies (1:200; A21428; Invitrogen; Thermo Fisher Scientific, Inc.) or with Alexa 488-conjugated secondary antibodies (1:200; A11008; Invitrogen; Thermo Fisher Scientific, Inc.) for $1 \mathrm{~h}$ at RT in the dark. Immunofluorescent images were examined and analyzed using the Olympus FV1000 confocal system (Olympus Corporation, Tokyo, Japan). Protein expression levels were determined using the mean fluorescence intensity values of the samples with the FV10 ASW 1.7 software (Olympus Corporation).

Statistical analysis. The values are expressed as the mean \pm standard deviation. Statistical analyses were performed using analysis of variance and Student's $t$-test. $\mathrm{P} \leq 0.05$ was considered to indicate a statistically significant difference. All statistical analyses were performed using the SPSS 17.0 software package (SPSS, Inc., Chicago, IL, USA).

\section{Results}

Differentially expressed lncRNA following exposure to cisplatin. Using ncFANs, several differentially expressed lncRNAs were identified. The increasing numbers of differentially expressed lncRNAs were showed in HepG2 cells as the exposure time for cisplatin increased. There were 6 , 26 and 86 differentially expressed lncRNAs identified after treatment for 12, 24 and $48 \mathrm{~h}$, respectively. In all these three time points, four lncRNAs were differentially expressed. They were RP11-134G8.8, RP11-612B6.2, RP11-363E7.4 and RP1-193H18.2 (Fig. 1A). RP11-134G8.8 and RP11-363E7.4 were upregulated after treatment with cisplatin, whereas RP11-612B6.2 and RP1-193H18.2 were downregulated.

lncRNA-gene co-expression network and functional enrichment. After calculating the PCC between each continuous differentially expressed lncRNAs and all genes, significant co-expressed genes were obtained for these differentially expressed lncRNAs. Then, the lncRNA-gene co-expression network was constructed, in which nodes were lncRNAs and genes. IncRNAs and genes were connected if they were significantly co-expressed (Fig. 2A). Some genes were found to be co-expressed with only one lncRNA,
A

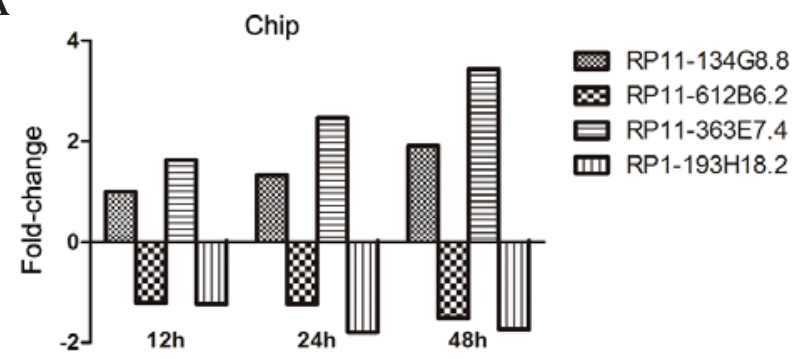

B

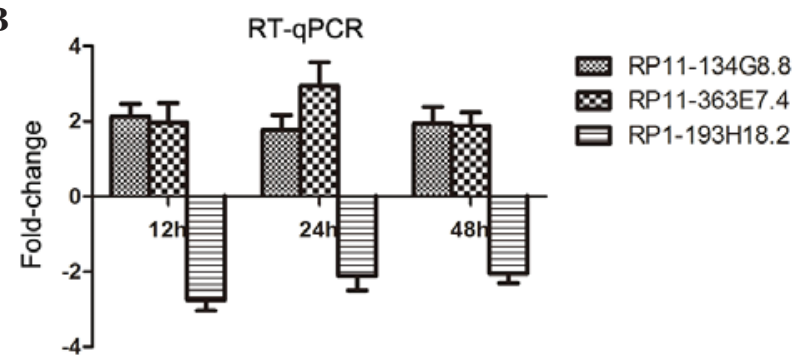

Figure 1. Differentially expressed lncRNAs in HepG2 cells after cisplatin treatment. (A) Fold-change value of four continuous differentially expressed lncRNAs at three time points by microarray analysis. (B) Three continuous differentially expressed lncRNAs at three time points were detected by RT-qPCR. RT-qPCR, reverse transcription-quantitative polymerase chain reaction; lncRNA, long noncoding RNA.

whereas other genes were co-expressed with more than one lncRNA. Functional annotation of the corresponding co-expressed genes was implemented to explore the potential function of these lncRNAs by using the DAVID 6.7 tool. The results demonstrated that they were annotated in some well-documented cancer-related pathways (for example, the p53 signaling pathway and the mitogen-activated protein kinase signaling pathway) (Table I). Particularly, some genes were co-expressed with three lncRNAs (red background in the center of Fig. 2A) and they were annotated into the p53 signaling pathway (Fig. 2B).

lncRNA validation. In order to confirm the four predicted lncRNAs, qPCR was performed at three time points after cisplatin exposure (12, 24 and $48 \mathrm{~h})$. GAPDH served as an internal control. The results in Fig. 1B show the standardized fold-changes in lncRNA expression in HepG2 cells incubated with cisplatin for different periods of time. After 12, 24 and $48 \mathrm{~h}$ of incubation, cisplatin significantly decreased the RP1-193H18.2 level by 2.75, 2.11 and 2.06-fold, respectively. RP11-134G8.8 expression increased by 1.77-2.13-fold, and the RP11-363E7.4 level was also upregulated by 1.89-2.94-fold. These trends in IncRNA expression are consistent with the microarray results. However, RP11-612B6.2 lncRNA was not amplified (data not shown).

Cell cycle test. To evaluate HepG2 cell proliferation, cell cycle was analyzed by flow cytometry. Flow cytometric analysis showed that the percentage of cells in the G0/G1 phase was significantly lower in HepG2 cells with cisplatin treatment for $24 \mathrm{~h}$ than in HepG2 cells under normal culture conditions (5.46 \pm 0.99 vs. $63.62 \pm 1.06, \mathrm{P}<0.01)$, whereas the percentage of 
$\mathbf{A}$

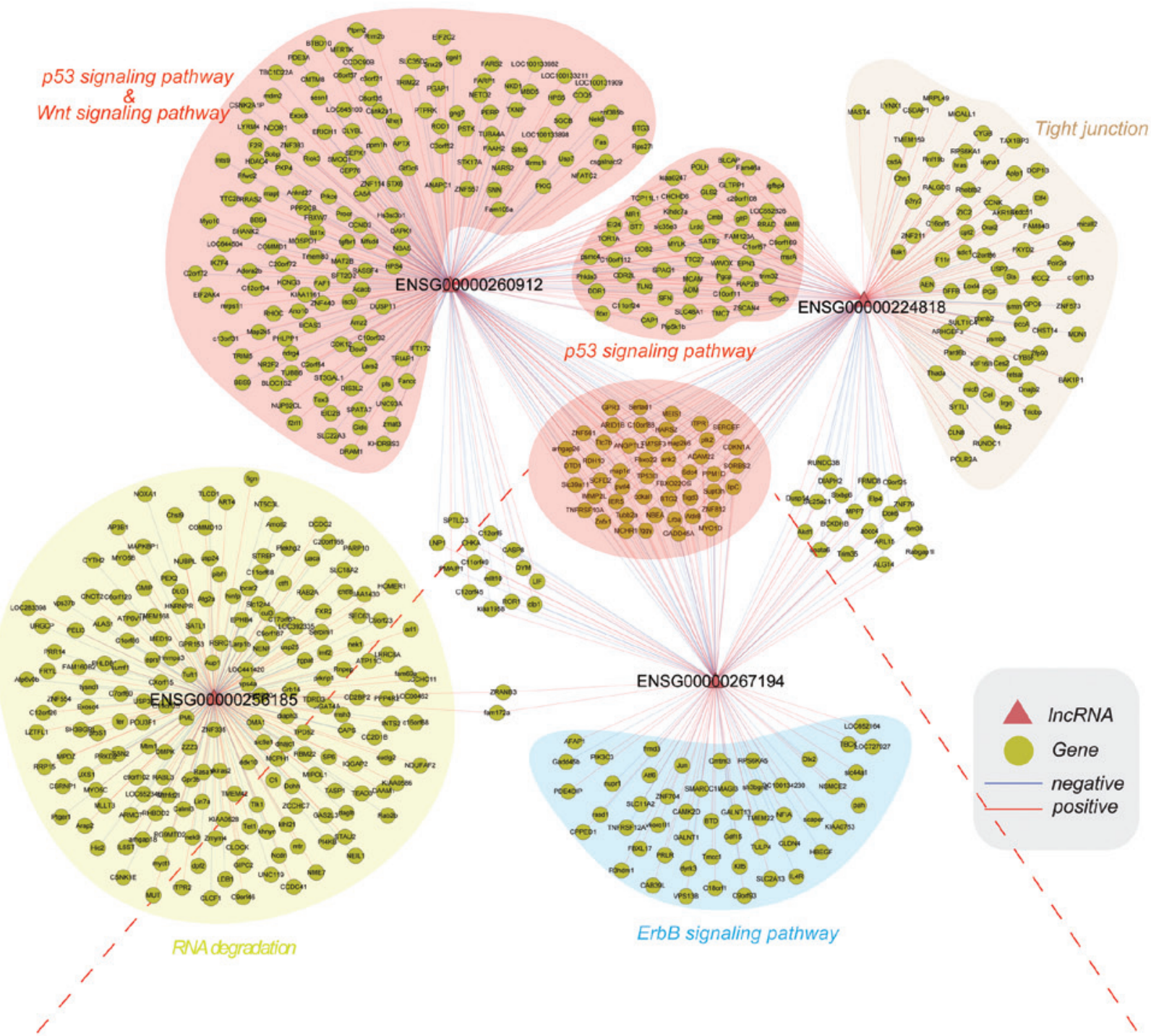

B

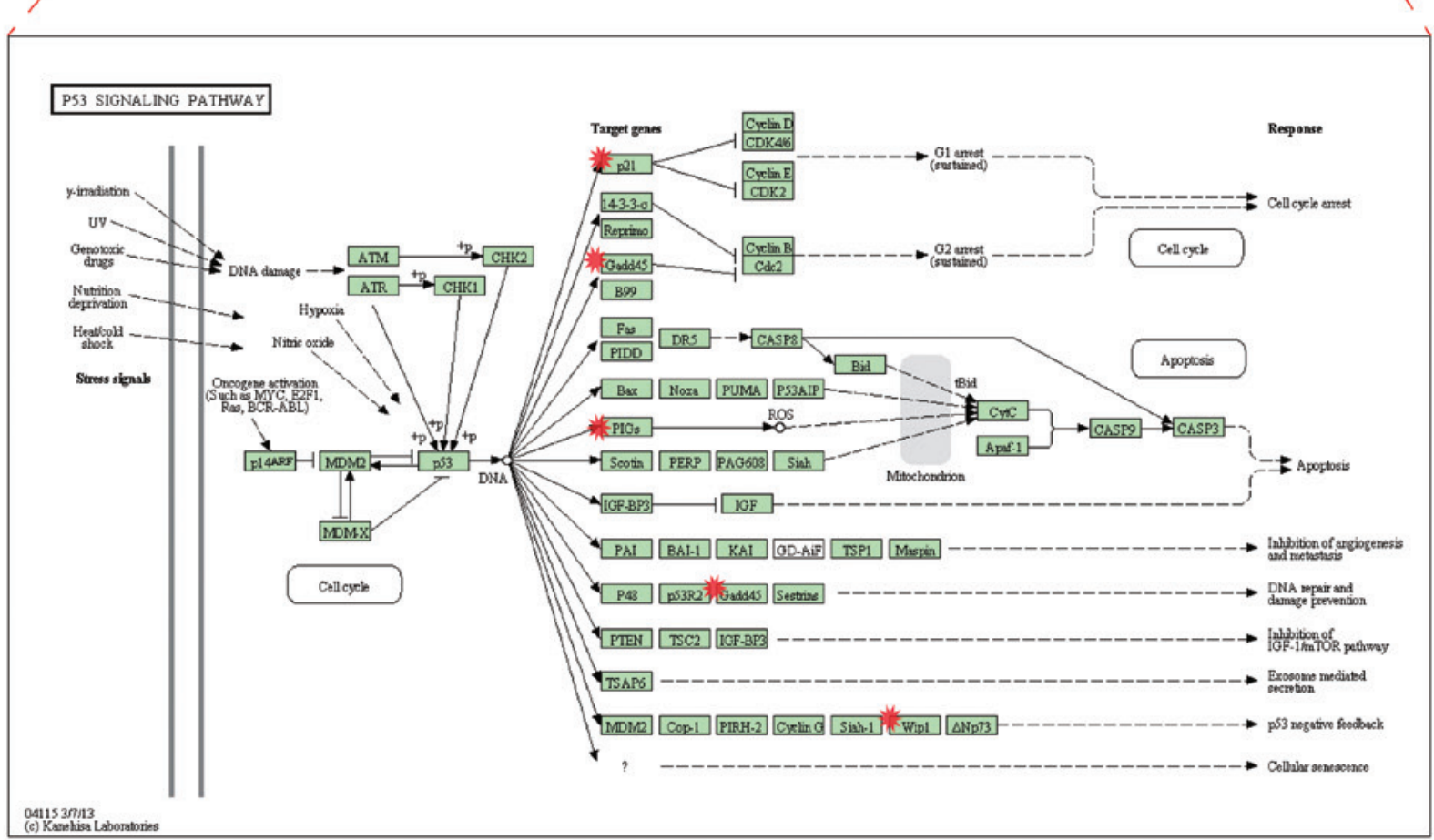

Figure 2. Functional investigation of lncRNAs. (A) lncRNA-gene co-expression network. Nodes were lncRNAs (triangles) and genes (circles). lncRNAs and genes were connected if they were significantly co-expressed. Red and blue lines represent positive and negative co-expressed associations, respectively. Different background color represents different pathways where the gene annotated. (B) p53 signaling pathway. Red marks represent the genes that were co-expressed with lncRNAs. lncRNA, long noncoding RNA. 


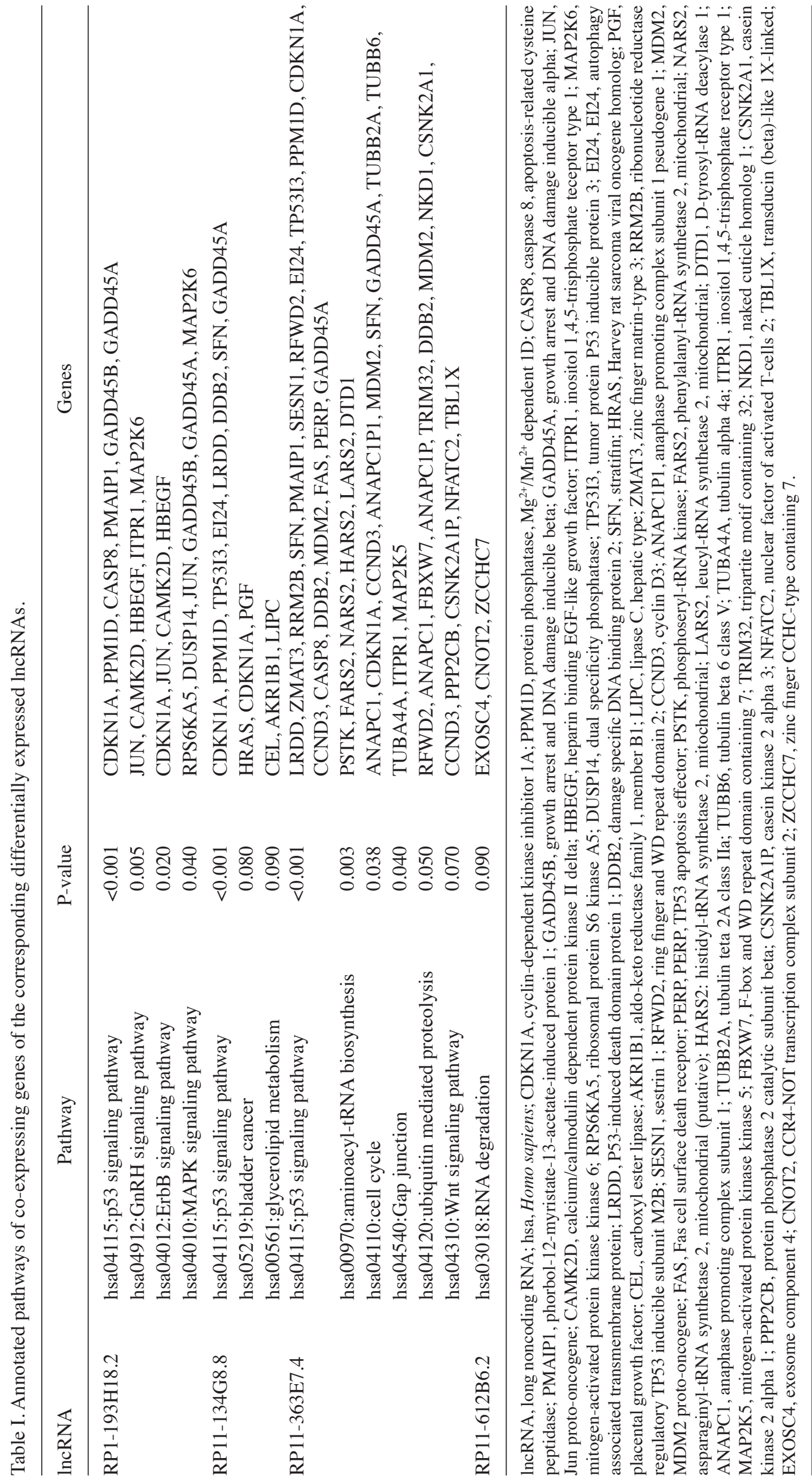


A

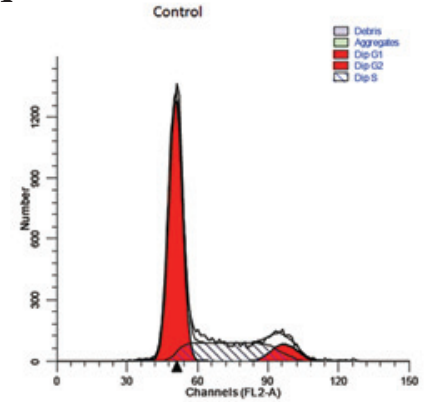

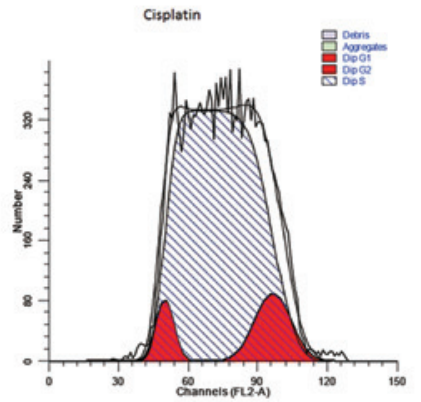

B

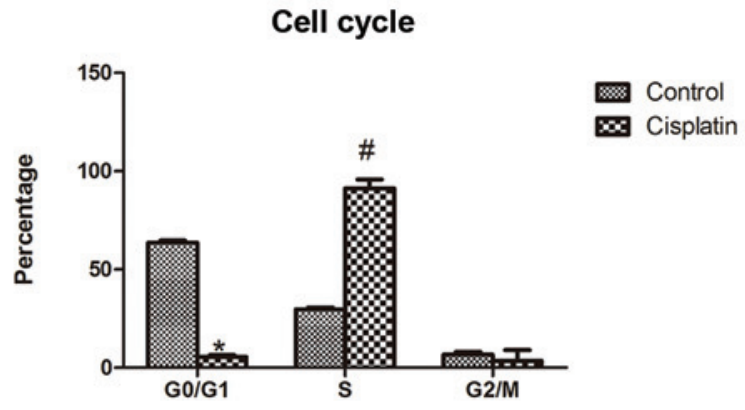

Figure 3. Effect of cisplatin on the cell cycle of HepG2 cells. (A) Flow cytometry analysis of the cell cycle. (B) Accumulated percentages of HepG2 cells were observed in the $\mathrm{S}$ phase after $24 \mathrm{~h}$ of cisplatin treatment $(7 \mu \mathrm{M})$, compared with the control group (untreated HepG2 cells). The results represent the mean \pm standard deviation of the values obtained in three independent experiments. Statistical significance was calculated using the Student's $t$-test. ${ }^{* *} \mathrm{P}<0.01$ vs. the control group.

A Control Cisplatin(12h) Cisplatin(24h)

B
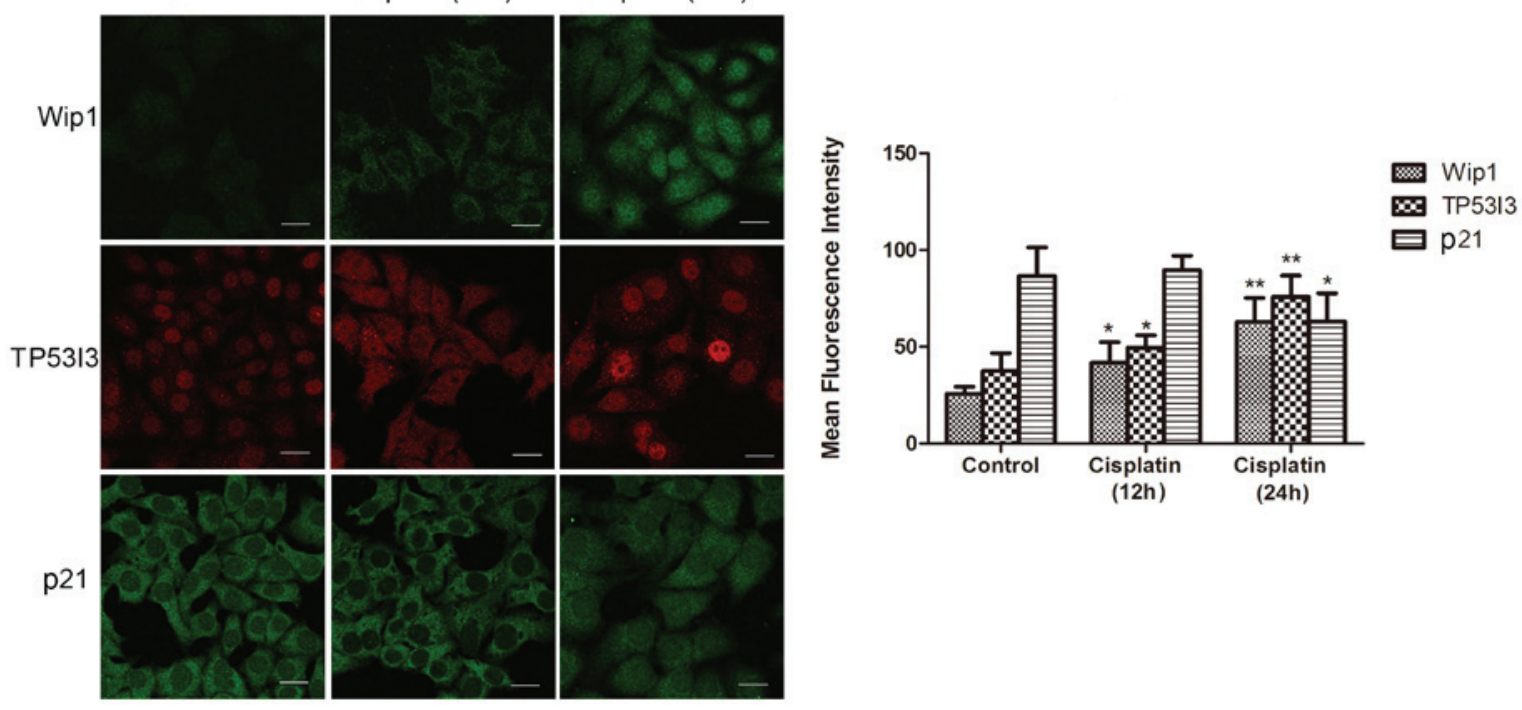

Figure 4. Effect of cisplatin on Wip1, TP53I3 and p21 expression. (A) Wip1, TP53I3 and p21 expression was observed by immunofluorescence staining (B) Wip1, TP53I3 and p21 expression was quantified by their mean fluorescence intensity values. Control: untreated HepG2 cells. Cisplatin: cisplatin treatment $(7 \mu \mathrm{M})$ for 12 or $24 \mathrm{~h}$ (scale bar $=20 \mu \mathrm{m}$ ). The results represent the mean \pm standard deviation of the values obtained in three independent experiments. Statistical significance was calculated using analysis of variance. ${ }^{*} \mathrm{P}<0.05,{ }^{* *} \mathrm{P}<0.01$ vs the control group. TP53I3, tumor protein p53 inducible protein 3 ; Wip1, wild-type p53-induced phosphatase 1.

cells in the $\mathrm{S}$ phase was significantly higher in the cisplatin treatment group than in untreated HepG2 cells $(91.15 \pm 4.59$ vs. $29.73 \pm 0.95, \mathrm{P}<0.01)$. No significant difference was noted in the percentage of cells in the $\mathrm{G} 2 / \mathrm{M}$ phase in the two groups of cells $(3.39 \pm 5.48$ vs. $6.65 \pm 1.42, \mathrm{P}>0.05)$. This result showed that cisplatin induced the arrest in the $\mathrm{S}$ phase of the cell cycle (Fig. 3).

Immunofluorescence staining. To evaluate the co-expression genes of these lncRNAs in HepG2 cells after cisplatin treatment, three genes (TP53I3, CDKN1A and PPM1D) were chosen. Immunofluorescence was carried out for their proteins: TP53I3, p21 (CDKN1A) and Wip1 (PPM1D). The results showed that the expression level of TP53I3 and Wip1 was upregulated in the cisplatin group compared with that of the control group. Inversely, compared with the control group, the expression level of p21 was downregulated in the cisplatin group (Fig. 4).

\section{Discussion}

It has been reported that lncRNAs possess important roles in gene regulation, though $\operatorname{lncRNAs}$ once were considered incapable of encoding proteins (19). Recently, the biological functions of lncRNAs have been received increased attention, and abnormal expression of some lncRNAs was found in HCC (1). However, only a few lncRNAs have been elucidated as targets of cancer diagnosis and therapy (19). Bioinformatics analyses or microarray have been helpful in lncRNAs research. In this study, four continuous differentially expressed lncRNAs (RP11-134G8.8, RP11-612B6.2, RP11-363E7.4 and RP1-193H18.2) were identified in cisplatin-treated HepG2 cells 
by analyzing microarray data. Furthermore, RP11-134G8.8, RP11-363E7.4 and RP1-193H18.2 were verified by qPCR. RP11-134G8.8 and RP11-363E7.4 were upregulated, while RP1-193H18.2 was downregulated. To explore the functions of the differentially expressed lncRNAs, we obtained and identified 57 significant co-expressing genes and pathways where the genes were involved in, such as the p53, ErbB, Wnt and gonadotropin-releasing hormone signaling pathways (Table I). Obviously, many co-expressing genes of the three lncRNAs (RP11-134G8.8, RP11-363E7.4 and RP1-193H18.2) were involved in the p53 signaling pathway, which showed the most significant difference among all pathways. Thus, after analyzing all data by bioinformatics, cell cycle was examined, and three key genes (CDKN1A, TP53I3 and PPM1D) in the p53 signaling pathway (20-23) were verified, which may be useful for exploring the deeply regulated mechanism of lncRNAs in HepG2 cells with cisplatin treatment.

CDKN1A/p21 is a cyclin-dependent kinase inhibitor involved in carcinogenesis by regulating the cell cycle progression at different phases $(24,25)$. As a famous downstream target of p53, changed expression of CDKN1A was found in various cancers and therapeutic processes (26-29). Fluoroquinolones were reported to have the ability to penetrate pancreatic tissue, and are usually associated with loss or downregulation of the CDK inhibitors p21/p27 as well as with the mutational inactivation of p53 (26). Significantly upregulated p21 at both the gene and protein levels was found in MCF-7 cells treated with Dillenia suffruticosa root dichloromethane extract, and the results suggested that the induction of G0/G1-phase cell cycle arrest in MCF-7 cells was achieved via the p53/p21 pathway (21). In human lung adenocarcinoma cells, it was found that the upregulation of the IncRNA HOTAIR contributes to cisplatin resistance, at least in part, through the regulation of p21 expression (30). It seems that protein/gene expression and cell cycle arrest are different in different cancers and conditions (17,22-26). In this study, CDKN1A was negatively regulated by RP1-193H18.2 and positively regulated by RP11-134G8.8 and RP11-363E7.4 at the gene level. The expression of p21 was downregulated after cisplatin treatment for $24 \mathrm{~h}$ at the protein level, contrarily to the upregulated $\mathrm{p} 21$ expression reported by $\mathrm{Qu}$ et al (27). However, it is easy to understand that the loss of p21 expression could be the result of apoptosis, which is induced by cisplatin. Additionally, there is a big interaction network between $\mathrm{p} 21$ and other regulatory factors; thus, further research on $\mathrm{p} 21$ is required.

TP53I3 is also induced by the tumor suppressor p53 and is thought to be involved in p53-mediated cell death $(23,31)$. p53-inducible gene 3 (PIG3) contributes to early cellular response to DNA damage and is a precursor of the apoptosis pathway that determines the fate of a cell in response to cellular stress (32). Our results showed that TP53I3 was negatively regulated by RP1-193H18.2 but positively regulated by RP11-134G8.8 and RP11-363E7.4 at the gene level, and PIG3 showed upregulated expression at the protein level. This could be the cell response to cisplatin at early times, trying to regulate the cell homeostasis (33). Indeed, the induction of p53 could also explain these results, since the p53 signaling pathway is involved in biological changes of HepG2 cells under cisplatin treatment.

As a gene in the p53 signaling pathway, PPM1D performs many physiological functions, including cell signaling, apoptosis and cell cycle progression $(22,34)$. The protein PPM1D is a member of the protein phosphatase 2C (PP2C) family of Ser/Thr protein phosphatases $(32,34)$. PPM1D is a stress-responsive PP2C phosphatase that plays a key role in stress signaling (35). In addition, it was suggested that PPM1D is associated with carcinogenesis $(36,37)$. It negatively regulates the DNA damage response through the dephosphorylation and inactivation of p53, ataxia telangiectasia mutated, p38 and checkpoint kinase 1/2 (38). In recent years, PPM1D was considered as a prognostic marker and potential therapeutic target in several cancers $(39,40)$. In this study, RP1-193H18.2 played a negative regulatory role for PPM1D at the gene level, while RP11-134G8.8 and RP11-363E7.4 were positive factors. As a result, upregulated protein levels of PPM1D were observed. This could be a response of the cell to the stress induced by cisplatin. Furthermore, it was found that PPM1D played an important role in promoting cisplatin resistance, and as a novel downstream target of Akt, PPM1D mediates its action of conferring cisplatin resistance to gynecological cancer cells (41). PPM1D could also be induced by p53 to maintain the homeostasis in cells (42). In addition, Cao et al reported that PPM1D plays a role in the cell cycle via p21 in dogs (43). Our research has identified opposite expression tends for $\mathrm{p} 21$ and PPM1D within short time of cisplatin treatment. However, more experiments must be designed for confirming if there is an association between them in humans.

Obvious cell cycle arrest at the $\mathrm{S}$ phase was induced by cisplatin in this study. These results are different from those of a previous report (27), despite the fact that a similar dose of cisplatin was used. The only difference is the exposure time to cisplatin. We hypothesize that cisplatin may induce $S$ phase arrest at only early times when cells are in the state of stress. This could be one of direct reactions of cells to the DNA duplicate damage induced by cisplatin. The change of cell cycle should be explicated for all relative factors.

In summary, the 1ncRNAs RP11-134G8.8, RP11-363E7.4 and RP1-193H18.2 were differentially expressed in HepG2 cells after cisplatin treatment. These lncRNAs may play an important role by regulating the expression of genes that are co-expressed with them. In addition, cell cycle arrest could be induced at the $\mathrm{S}$ phase when cells were treated with cisplatin for a short time. As a classic pathway, the p53 signaling pathway contributes to the effect of cisplatin and its induced resistance. Therefore, the lncRNAs RP11-134G8.8, RP11-363E7.4 and RP1-193H18.2 and their co-expressed genes, which annotated into the p53 signaling pathway, could be potential biomarkers for cisplatin treatment. This study will help to understand the lncRNAs functions in HepG2 cells under cisplatin treatment.

\section{Acknowledgements}

The present study was supported by the National Science Foundation of China (Beijing, China; grant no. 30873131) and and by the Jilin Province Development and Reform Commission (Jilin, China; grant no. 2016C023).

\section{References}

1. Hou JK, Huang Y, He W, Yan ZW, Fan L, Liu MH, Xiao WL, Sun HD and Chen GQ: Adenanthin targets peroxiredoxin I/II to kill hepatocellular carcinoma cells. Cell Death Dis 5: e1400, 2014. 
2. Yang X, Xie X, Xiao YF, Xie R, Hu CJ, Tang B, Li BS and Yang SM: The emergence of long non-coding RNAs in the tumorigenesis of hepatocellular carcinoma. Cancer Lett 360: $119-124,2015$

3. Marullo R, Werner E, Degtyareva N, Moore B, Altavilla G, Ramalingam SS and Doetsch PW: Cisplatin induces a mitochondrial-ROS response that contributes to cytotoxicity depending on mitochondrial redox status and bioenergetic functions. PLoS One 8: e81162, 2013.

4. Wang Y, Liu Y, Liu Y, Zhou W, Wang H, Wan G, Sun D, Zhang N and Wang Y: A polymeric prodrug of cisplatin based on pullulan for the targeted therapy against hepatocellular carcinoma. Int J Pharm 483: 89-100, 2015.

5. Ziko L, Riad S, Amer M, Zdero R, Bougherara H and Amleh A: Mechanical stress promotes cisplatin-induced hepatocellular carcinoma cell death. Biomed Res Int 2015: 430569, 2015.

6. Bose RN: Biomolecular targets for platinum antitumor drugs. Mini Rev Med Chem 2: 103-111, 2002.

7. Chu G: Cellular responses to cisplatin. The roles of DNA-binding proteins and DNA repair. J Biol Chem 269: 787-790, 1994

8. Galluzzi L, Senovilla L, Vitale I, Michels J, Martins I, Kepp O, Castedo $\mathrm{M}$ and Kroemer G: Molecular mechanisms of cisplatin resistance. Oncogene 31: 1869-1883, 2012.

9. Bonasio R and Shiekhattar R: Regulation of transcription by long noncoding RNAs. Annu Rev Genet 48:433-455, 2014.

10. Kung JT, Colognori D and Lee JT: Long noncoding RNAs: Past, present and future. Genetics 193: 651-669, 2013.

11. Shi D, Zheng H, Zhuo C, Peng J, Li D, Xu Y, Li X, Cai G and Cai S: Low expression of novel lncRNA RP11-462C24.1 suggests a biomarker of poor prognosis in colorectal cancer. Med Oncol 31: 31, 2014.

12. Sun M and Kraus WL: From discovery to function: The expanding roles of long noncoding RNAs in physiology and disease. Endocr Rev 36: 25-64, 2015

13. Fan Y, Shen B, Tan M, Mu X, Qin Y, Zhang F and Liu Y: Long non-coding RNA UCA1 increases chemoresistance of bladder cancer cells by regulating Wnt signaling. FEBS J 281: $1750-1758,2014$.

14. George J and Patel T: Noncoding RNA as therapeutic targets for hepatocellular carcinoma. Semin Liver Dis 35: 63-74, 2015.

15. Liao Q, Xiao H, Bu D, Xie C, Miao R, Luo H, Zhao G, Yu K, Zhao H, Skogerb $\varnothing$ G, et al: ncFANs: A web server for functional annotation of long non-coding RNAs. Nucleic Acids Res 39: W118-W124, 2011

16. Hou Z, Xu C, Xie H, Xu H, Zhan P, Yu L and Fang X: Long noncoding RNAs expression patterns associated with chemo response to cisplatin based chemotherapy in lung squamous cell carcinoma patients. PLoS One 9: e108133, 2014

17. Sui W, Yan Q, Li H, Liu J, Chen J, Li L and Dai Y: Genome-wide analysis of long noncoding RNA expression in peripheral blood mononuclear cells of uremia patients. J Nephrol 26: 731-738, 2013.

18. Huang da W, Sherman BT and Lempicki RA: Systematic and integrative analysis of large gene lists using DAVID bioinformatics resources. Nat Protoc 4: 44-57, 2009.

19. Ponting CP, Oliver PL and Reik W: Evolution and functions of long noncoding RNAs. Cell 136: 629-641, 2009.

20. Hall JR, Messenger ZJ, Tam HW, Phillips SL, Recio L and Smart RC: Long noncoding RNA lincRNA-p21 is the major mediator of UVB-induced and p53-dependent apoptosis in keratinocytes. Cell Death Dis 6:e1700, 2015.

21. Foo JB, Yazan LS, Tor YS, Wibowo A, Ismail N, How CW, Armania N, Loh SP, Ismail IS, Cheah YK and Abdullah R: Induction of cell cycle arrest and apoptosis by betulinic acid-rich fraction from Dillenia suffruticosa root in MCF-7 cells involved p53/p21 and mitochondrial signalling pathway. J Ethnopharmacol 166: 270-278, 2015.

22. Ma X, Han J, Wu Q, Liu H, Shi S, Wang C, Wang Y, Xiao J, Zhao J, Jiang J and Wan C: Involvement of dysregulated Wip1 in manganese-induced p53 signaling and neuronal apoptosis. Toxicol Lett 235: 17-27, 2015.

23. Flatt PM, Polyak K, Tang LJ, Scatena CD, Westfall MD, Rubinstein LA, Yu J, Kinzler KW, Vogelstein B, Hill DE and Pietenpol JA: p53-dependent expression of PIG3 during proliferation, genotoxic stress and reversible growth arrest. Cancer Lett 156: 63-72, 2000.
24. Gartel AL and Radhakrishnan SK: Lost in transcription: p21 repression, mechanisms and consequences. Cancer Res 65: 3980-3985, 2005.

25. Ando T, Kawabe T, Ohara H, Ducommun B, Itoh $M$ and Okamoto T: Involvement of the interaction between p21 and proliferating cell nuclear antigen for the maintenance of $\mathrm{G} 2 / \mathrm{M}$ arrest after DNA damage. J Biol Chem 276: 42971-42977, 2001.

26. Yadav V, Sultana S, Yadav J and Saini N: Gatifloxacin induces $\mathrm{S}$ and $\mathrm{G} 2$-phase cell cycle arrest in pancreatic cancer cells via p21/p27/p53. PLoS One 7: e47796, 2012.

27. Qu K, Lin T, Wei J, Meng F, Wang Z, Huang Z, Wan Y, Song S, Liu S, Chang H, et al: Cisplatin induces cell cycle arrest and senescence via upregulating P53 and P21 expression in HepG2 cells. Nan Fang Yi Ke Da Xue Xue Bao 33: 1253-1259, 2013.

28. Wang C, Chen Z, Ge Q, Hu J, Li F, Hu J, Xu H, Ye Z and Li LC: Up-regulation of p21 (WAF1/CIP1) by miRNAs and its implications in bladder cancer cells. FEBS Lett 588: 4654-4664, 2014

29. Zhang H, Zhang X, Ji S, Hao C, Mu Y, Sun J and Hao J: Sohlh2 inhibits ovarian cancer cell proliferation by upregulation of p21 and downregulation of cyclin D1. Carcinogenesis 35: 1863-1871, 2014

30. Liu Z, Sun M, Lu K, Liu J, Zhang M, Wu W, De W, Wang Z and Wang R: The long noncoding RNA HOTAIR contributes to cisplatin resistance of human lung adenocarcinoma cells via downregualtion of p21 (WAF1/CIP1) expression. PLoS One 8: e77293, 2013

31. Contente A, Dittmer A, Koch MC, Roth J and Dobbelstein M A polymorphic microsatellite that mediates induction of PIG3 by $\mathrm{p} 53$. Nat Genet 30: 315-320, 2002.

32. Guan X, Liu Z, Wang L, Wang LE, Sturgis EM and Wei Q: Functional repeats (TGYCC)n in the p53-inducible gene 3 (PIG3) promoter and susceptibility to squamous cell carcinoma of the head and neck. Carcinogenesis 34: 812-817, 2013.

33. Li B, Shang ZF, Yin JJ, Xu QZ, Liu XD, Wang Y, Zhang SM, Guan $\mathrm{H}$ and Zhou PK: PIG3 functions in DNA damage response through regulating DNA-PKcs homeostasis. Int J Biol Sci 9: 425-434, 2013

34. Shaltiel IA, Aprelia M, Saurin AT, Chowdhury D, Kops GJ, Voest EE and Medema RH: Distinct phosphatases antagonize the p53 response in different phases of the cell cycle. Proc Natl Acad Sci USA 111: 7313-7318, 2014.

35. Lowe J, Cha H, Lee MO, Mazur SJ, Appella E and Fornace AJ Jr: Regulation of the Wip1 phosphatase and its effects on the stress response. Front Biosci (Landmark Ed) 17: 1480-1498, 2012.

36. Wang W, Zhu H, Zhang H, Zhang L, Ding Q and Jiang H: Targeting PPM1D by lentivirus-mediated RNA interference inhibits the tumorigenicity of bladder cancer cells. Braz J Med Biol Res 47: 1044-1049, 2014.

37. Li GB, Zhang XL, Yuan L, Jiao QQ, Liu DJ and Liu J: Protein phosphatase magnesium-dependent 1delta (PPM1D) mRNA expression is a prognosis marker for hepatocellular carcinoma. PLoS One 8: e60775, 2013.

38. Yagi H, Chuman Y, Kozakai Y, Imagawa T, Takahashi Y, Yoshimura F, Tanino K and Sakaguchi K: A small molecule inhibitor of p53-inducible protein phosphatase PPM1D. Bioorg Med Chem Lett 22: 729-732, 2012.

39. Tan DS, Lambros MB, Rayter S, Natrajan R, Vatcheva R, Gao Q, Marchio C, Geyer FC, Savage K, Parry S, et al: PPM1D is a potential therapeutic target in ovarian clear cell carcinomas. Clin Cancer Res 15: 2269-2280, 2009.

40. Peng TS, He YH, Nie T, Hu XD, Lu HY, Yi J, Shuai YF and Luo M: PPM1D is a prognostic marker and therapeutic target in colorectal cancer. Exp Ther Med 8: 430-434, 2014.

41. Ali AY, Kim JY, Pelletier JF, Vanderhyden BC, Bachvarov DR and Tsang BK: Akt confers cisplatin chemoresistance in human gynecological carcinoma cells by modulating PPM1D stability. Mol Carcinog 54: 1301-1314, 2015.

42. Park HK, Panneerselvam J, Dudimah FD, Dong G, Sebastian S, Zhang J and Fei P: Wipl contributes to cell homeostasis maintained by the steady-state level of Wtp53. Cell Cycle 10: 2574-2582, 2011.

43. Cao R, Zhang J, Zhang M and Chen X: PPM1D regulates $\mathrm{p} 21$ expression via dephoshporylation at serine 123. Cell Cycle 14: 641-647, 2015. 